\title{
The Nature of the Heisenberg-von Neumann Cut: Enhanced Orthodox Interpretation of Quantum Mechanics
}

\author{
Ashok Narasimhan $^{1}$ - Deepak Chopra ${ }^{2}$ - Menas C. Kafatos ${ }^{3,4}$ (D) \\ Received: 31 January 2019 / Accepted: 28 March 2019 / Published online: 27 April 2019 \\ (C) The Author(s) 2019
}

\begin{abstract}
We examine the issue of the Heisenberg-von Neumann cut in light of recent interpretations of quantum eraser experiments which indicate the possibility of a universal Observer outside space-time at an information level of existence. The delayed-choice aspects of observation, measurement, the role of the observer, and information in the quantum framework of the universe are discussed. While traditional double-slit experiments are usually interpreted as indicating that the collapse of the wave function involves choices by an individual observer in space-time, the extension to quantum eraser experiments brings in some additional subtle aspects relating to the role of observation and what constitutes an observer. Access to, and the interpretation of, information outside space and time may be involved. This directly ties to the question of where the Heisenberg-von Neumann cut is located and what its nature is. Our model is an interpretation which we term the Enhanced Orthodox Interpretation of Quantum Mechanics. It does not contradict the standard orthodox interpretation, but we believe it extends it by approaching von Neumann's work in a new way. The Enhanced Orthodox Interpretation accepts the presence of a universal Observer, retaining the importance of observation augmented by the role of information. There is a possibility that individual observers making choices in space and time are actually aspects of the universal Observer, a state masked by assumptions about individual human minds that may need further development and re-examination.
\end{abstract}

Keywords Quantum mechanics · Copenhagen interpretation · Heisenberg-von Neumann cut · Enhanced orthodox interpretation · Observer $\cdot$ Measurement theory $\cdot$ Collapse of the wave function $\cdot$ Information

\section{Introduction}

The Copenhagen Interpretation of Quantum Mechanics (CI) was proposed initially a century ago by Niels Bohr and Werner Heisenberg, with further developments by them, Wolfgang Pauli, Max Born, and others (cf. Kafatos and Nadeau 2000; Cassidy 2008). In the CI, the act of observation plays a fundamental role in explaining the nature of reality. The

Menas C. Kafatos

kafatos@ chapman.edu

1 Associated Faculty, California Institute for Integral Studies, 1453 Mission Street, San Francisco, CA 94103, USA

2 Clinical Professor of Medicine, University of California at San Diego, San Diego, CA 92093, USA

3 CEESMO, Chapman University, One University Drive, Orange, CA 92866, USA

4 Korean Academy of Science and Technology, Seongnam-si, South Korea so-called collapse of the wave function denotes the process through which quantum possibilities become actualities in the "real" world of experience. The original Copenhagen school was later enriched by the work of John von Neumann (1995) and is now often referred to as the Orthodox Interpretation (heretofore, and in agreement with Stapp (2007, 2009, 2017), we can also refer to it as standard quantum mechanics).

In this standard Orthodox QM, there are two main types of processes as developed by von Neumann. Process 1 presents non-linear aspects of QM, wherein a "collapse" takes place upon observation, the actual outcome of which is unknown a priori. Process 2 is the predictable, linear time evolution of the wave function through the Schrödinger equation. The exact nature of collapse and what causes it remain controversial topics to this day, but in the Orthodox Interpretation of QM, it is taken for granted that the act of observation is essential for the collapse of the wave function: out of a large set of possible outcomes, observation confers reality on the specific outcome of a measurement performed in the laboratory, such as determining the direction of the spin of a particle. 
Even if the experimental process is automated, the participation of an observer remains essential, because we inhabit a participatory universe (Wheeler 1981). The mind that plans, executes, and undertakes an observation is termed here the (human) local observer, denoted by lower case $o$. This mind cannot be extracted from what is being observed. Observer $o$ sets up the quantum observation or "probing action" to ask a question of Nature, which responds with its answer (Process 1) at some initial time. This answer is recorded by the measuring apparatus. $\mathrm{S} / \mathrm{He}$ observes the outcome, which becomes an actuality, according to linear time evolution, or Process 2. The result can be recorded at a later time as a defined outcome. In the CI, observation causes the particular form of Nature's answer-e.g., particle or interference pattern in the double-slit experiment, as described in many references (e.g., Donati et al. 1973; Kafatos and Nadeau 2000; Stapp 2007).

In the context of the $\mathrm{CI}$, the crucial question arising from the double-slit experiment is whether the measurement requires a conscious observer. In Orthodox QM, the answer is yes. A conscious observer is required because information itself is relevant in quantum experiments only through a human observer. Information, measurement, and the setting up of experiments are intertwined human activities. The state of information without a mind to give it meaning is highly problematic. The relevant issues are explored below. We note that it was Stapp who has been the main consistent advocate of the collapse agent being a sentient animal, not necessarily human, although these ideas can be traced to the original CI.

\section{The Cut}

The two domains of Nature accepted in modern physics are quantum and classical, each obeying its own rules and seemingly contradictory in many ways. The Heisenberg cut (Atmanspacher 1997; Stapp 2017 and previous works) is the hypothetical boundary between these two domains. Below the cut occur quantum events, while above the cut, in the world occupied by human observers and experimenters, there are three general aspects at work, namely the information gained through doing experiments, the knowledge accumulated via this information, and the conscious awareness of the scientist. (Speaking broadly, the same three things - information, knowledge, and conscious awareness - are common to all experiences, not simply science.)

In Heisenberg's view, below the cut, everything is governed by the QM wave function, whereas above the cut a classical description applies. The Heisenberg cut as first proposed is a hypothetical level and may not even exist. von Neumann went on to ask where in the human body-mind might the cut actually be located. This focus on the human dimension was useful in terms of sensory input and what the mind makes of "events," with von Neumann insisting that the physical and the psychological must each be given its own significance. Thus, tracing a photon in the double-slit experiment to the eye of the observer and following its course in the brain, down to the most precise quantum interactions, will not tell the whole story. At some point, the information recorded in the experiment must be interpreted by the mind, through non-physical channels. The cut is therefore a psycho-physical boundary, not a purely physical one.

In order to evaluate the cut ontologically, myriad assumptions come into play. In the decades during which the doubleslit experiment has been considered and reconsidered, many implicit assumptions made by $\mathrm{CI}$ and later Orthodox CI have shown the need for revisiting in serious consideration. Among all the relevant factors associated with the various setups of the double-slit experiment, we can point to the following, which are all implicitly present:

(a) A relatively simple experimental system is proposed with two slits and the ability to pass photons through the slits.

(b) A measuring system is set up that can observe which slit the photon passes through.

(c) A human being consciously decides to observe or not observe the experiment.

(d) The observation by the human being takes place at a point in time within the space-time co-ordinates of the experiment.

(e) The consequence of the observation is that when the "which" path (i.e., the known path that quanta follow, revealing their particle nature, or when we know which slit a particular quantum went through) is observed, the recorded end result on the media indicates quanta localized in space and time, what we term particles. When the human decides not to observe, the recorded end result is an interference pattern ("scrambled" path), indicating a wave nature (Narasimhan and Kafatos 2016).

(f) The act of deciding to observe or not, and then making the observation or not, requires the will of the conscious human to be a factor. In fact, this is the determining factor in whether the wave function collapses or not.

All QM events occur in, and are subject to, space-time and its laws. Behind the array of factors we have just listed, the original CI makes one crucial blanket assumption, that the entire system of measurement and human observer are in ordinary space-time, and should conform to experiencing a classical physics world. We challenge this by arguing that many of the QM events actually happen in "not-space-time," or beyond a classical world confined in space-time. In other words, consciousness is rooted in a dimensionless space that is not subject to conventional limitations in space-time. This possibility expands the observer's role in the double-slit experiment, making her/him a participant in a universal Observer. Our challenge is based on the wellverified results of a class of quantum experiments that extend the traditional CI framework of the double-slit experiment.

One should note that the interpretations of QM which do not recognize the reality of collapsing the wave function, e.g., the De Broglie-Bohm and the many world's interpretation, do not recognize the need for the Heisenberg-von Neumann cut. 
In addition, without going into details, there are other views which argue that what actually causes the collapse of the wave function is the act of physical measurement (the reader is directed to a good summary of various interpretations of quantum mechanics in Wikipedia listed in the References). This act can be carried out automatically and does not need a human observer, wherein the position-momentum uncertainty relation makes it impossible to determine which slit the photon passes through without disturbing the photon and destroying the interference pattern. The impossibility of not disturbing the photon through the act of observing it means that measurement (which we can simply define for our purposes here as the determination by the observer of the availability of which-path information) by a human observer cannot be the cause of the collapse of the wave function. Another school of thought attributes the collapse to an environment (presumably macroscopic) that surrounds the quantum experiment (Zurek 2003). However, since the environment can be considered as a classical system, this approach requires further work - it is not clear how one gets around the issue of making decisions in a classical setting about observations of quantum systems in the laboratory.

\section{Observation, Information, and Collapse of the Wave Function}

Now let us look in more detail at modifications of double-slit experiments spanning more than two decades (e.g., Scully and Drühl 1982; Zajonc et al. 1991; Walborn et al. 2002) that reproduce the double-slit experiment but use quantum methodologies with two ends in mind: First is to eliminate the need for any physical measurement that can scatter or introduce large uncontrolled phase factors. These would cause a disruption of the wave and collapse it. Second is the use quantum effects that allow for erasing of which-path information. We refer to one such experiment by Kim et al. (2000), which has been described in great detail before (Narasimhan and Kafatos 2016; Kafatos and Narasimhan 2018).

In short, this experiment involved two possible types of paths, one revealing the particle aspect ("which-path"), the other revealing the wave aspect but contrary to the doubleslit experiment, the human observer is taken out of the picture as no actual choice is made.

In accordance with our previous interpretations of this experiment (Narasimhan and Kafatos 2016; Kafatos and Narasimhan 2018), let us clarify some terms. At an ontological level, information is defined as "what is conveyed or represented by a particular arrangement or sequence of things." Information is structured such that an observer can draw conclusions from it, as in the availability of which-path information. In our view, information is differentiated from noise. If nothing is "conveyed or represented," then there is no information, only noise, which refers to the lack of specific, useful data tying to the quantum system.

For our purposes here, we focus on one kind of information, namely what we referred to above as "which-path" information. When something is "conveyed or represented" about the whichpath a quantum, in the Kim et al experiments a photon, has traveled, there is information. When there is nothing conveyed or represented about a which-path, there is no information. In our view, this is a critical distinction, since we say that what causes the collapse of the wave function is not the act of measurement itself but the availability of information. However, it should be pointed out that this type of retrocausality can not be used to send information e.g. about roulette wheel outcomes.

To recapitulate, observation is the availability of information to an observer. Measurement is the determination by the observer of the availability of which-path information. The observer is conscious, since $\mathrm{s} / \mathrm{he}$ needs to distinguish between information and noise, a conscious act. An unconscious entity or machine cannot make this differentiation because such a device would have no awareness of the different results being measured. In our previous work, we discussed the specific roles of humans in the experimental process. The "Grandfather" is the human who initially sets up the experiment, while the "Granddaughter" is the human who returns at some point in the future (practically $\sim$ infinity in the future) to observe the actual recordings laid down on the recording media. This means that the presence of a human observer is not necessarily required at the time of measurement, which does not in any way change the recorded results of the experiment.

The conclusions that we draw from the Kim et al. (2000) experiment are as follows: It is not the "act of measurement" that causes the collapse of the wave function. The act of measuring when which-path information is available is the same as the act of measuring where the which-path information has been erased, whereupon the interference pattern is recovered and not available to the human observer. The Grandfather and Granddaughter play no role in the process of the photon's traveling. They do not need to be present making active willful observations when the experiment is running. It can be set up in the distant past and the results observed in the distant future. So the conscious decision by a human observer to make a measurement and observe the which-path information (or not) has no impact on the results, thus eliminating the human observer as the causative agent in the collapse of the wave function.

Again, it is the availability of information (specifically the availability of which-path information) that causes the collapse of the wave function. The availability of information requires us to ask, "Available to whom?" the answer is "an observer," because if there is no observer, there is no one and nothing to register/observe the availability of the information. Without an observer, there can be no branching of the two possible results of the experiment (i.e., recording either a particle of an interference pattern). The ability to distinguish 
between availability (or not) of which-path information requires a conscious agent, regardless of when it enters the process.

We believe that the delayed-choice quantum eraser experiments (e.g., Kim et al. 2000) which have been carried out and described in great detail before, point to subtle aspects that the traditional double-slit experiment may miss. For example, one consequence coming into play is that the conscious observer is not required to be human. When human observers are taken out of the picture, a collapse still occurs. The Granddaughter's presence is only required at the time of looking at the recording at time $\sim$ infinity.

\section{The Nature and Location of the Cut}

We note here that there is really no "moment" of measurement. The results of the experiment show that the measurement of which-path information made at $t=1$ resulting in a photon being recorded, gets changed to wave interference being recorded when the which-path information is erased at $t=1+8 \mathrm{~ns}$ on its entangled twin (produced in a beam splitter, where entanglement means that the two photons (particles) are never apart). In that event, the entangled twin registers as wave interference. The essence of these quantum delayedchoice experiments is that there is apparent retrocausality, where a measurement in the future $(t=1+8 \mathrm{~ns})$ changes the measurement made in the past $(t=1)$.

Ontologically, causality is based on the classical framework of space-time, which holds that time (unlike space) is unidirectional and that events in the past can only affect events in the future, not the other way around. However, we are faced with experimental results that indicate that this is exactly what is happening. If retrocausality exists, which is incompatible with the classical space-time framework, then we need to admit the possibility that observation is occurring in a not-space-time (we will call it NST) frame of reference. Note that we do not say "outside" space-time, since "outside" is a term rooted in the classical (mis)conception that space has an exterior and interior.

For the present one can only speculate about the nature of NST, but with the validity of delayed-choice quantum eraser experiments, we are obliged to consider how NST affects our assumptions about the results of the double-slit experiment, and more generally how our assumptions about reality are affected.

In a sense, the CI/von Neumann/Stapp orthodox interpretation is a special case of the availability of information when a human observer is an integral part of the picture. The assumption that everything surrounding the experiment takes place in classical space-time follows logically from the need for a human observer, since classical space-time is our own domain. Outside this particular experimental setup, the general case explores the possibility that the entire process occurs in both space-time and not-space-time (NST). Space-time and notspace-time can be viewed as complementary aspects of the complete reality. We believe that the complementarity between the classical and quantum "regions" is important for being able to fit the experimental evidence into a valid conceptual framework (see also Herzog et al. 1995). Another way of saying it is that the measurement is not in space-time. Having already removed any temporal requirement, a requirement involving space must also be removed (i.e., there is no specific place at which the double-slit experiment must be performed).

Since the cut is involved at the point of measurement, we can say that in this ontological approach the cut is also not in spacetime. In short, both are non-local. Non-locality is a well-verified phenomenon involving any event not conforming to the set limits of space-time. As we saw, it has always been assumed that the cut takes place at the moment of measurement. Human reality is based on our perception of space-time, so naturally a measurement is inextricably linked to a moment when it takes place. Similarly, we have assumed that the cut takes place in a position in space. Thus von Neumann offered a thought experiment to investigate where the cut may be located in the path from the eye to the brain, asking whether the cut is in the retina or the optic nerve or the cells of the visual cortex. All of these locations are necessarily in physical space.

According to Heisenberg, the dividing line between the system to be observed and the measuring apparatus is immediately defined by the nature of the problem. This obviously signifies no discontinuity in the physical process; Nature and the experiment are seamlessly connected, the one posing a question, the other instantaneously presenting an answer in the form of some observed phenomenon. For this reason, there must, within limits, exist complete freedom in choosing the position of the dividing line. This line of reasoning led von Neumann to declare that the cut was nowhere. But then if it is nowhere, it must be in some sense everywhere. The problem is that we are still (naturally) thinking in terms of "where," which is rooted in conventional space-time. If the cut is not in space-time, as suggested in our approach, this tenet is fully consistent with the inability to locate the cut in the classical approach. And since the cut takes place in the observer, this implies that the observer is not in space-time, either.

However, the observer making the measurement not in space-time has consequences that occur in space-time, i.e., the appearance of the recording on the media and the human observer who sees it. In contrast to traditional CI, our case is more general once the human observer as an active causative agent is taken out. We believe that Orthodox CI is a special case, applicable when the entire process is believed to occur in ordinary space-time. Our more general case explores the possibility that the entire process occurs in both space-time and not-space-time, the two being complementary aspects of the complete reality.

By implication, there must be some mechanism that allows the consequences of the observer and cut in not-space-time to appear in space-time. Such a mechanism permits "leakage" of information between not-space-time and space-time. Leakage occurs when 
processes move between two complementary regions. In accord with non-locality, the whole reality governs classical and quantum regions without rigid barriers or distinctions between them.

\section{Final Thoughts}

In the CI/Orthodox view, as noted above, observers are not defined but are generally assumed to be human. That seems to be a questionable anthropocentric viewpoint. A broader and logical view that we will explore in future works is that our Enhanced Orthodox Interpretation assumes that the observer is real; in fact, all types of observers, human and non-human, are real but also not separate from each other, they are aspects of one universal existence. One can posit that an animal (say a dog) with the right equipment could also observe a QM binary choice (perhaps up or down spin is connected to a food choice). Would such a dog observation cause collapse of the wave function? In CI, this possibility is not even considered but in principle would be possible, bringing up a host of other questions. In our approach, such dilemmas do not arise, because it is the availability of information to the observer, and not the presence of a human observer making measurements that is the causative factor for the collapse of the wave function. As pointed out to us, what we are suggesting in the present work might also be on the level of information processing in the brain that enable integrating various quasi-independent observers in the human mind, perhaps related to dissociative phenomena and subliminal information. These are worth exploring in the future.

\section{Conclusions}

In the present work, we examine the nature and location of the Heisenberg-von Neumann cut in light of recent interpretations of a class of quantum eraser experiments, indicating the possibility of a universal Observer, outside of what we perceive as classical space-time, at an information level of existence. The delayed-choice aspects of observation, measurement, the role of the observer, and information in the quantum universe provide additional insights that traditional double-slit experiments may miss. The extension of the Orthodox CI framework relates to issues about where the Heisenberg-von Neumann cut is located and what is its nature. Our work does not contradict the standard Orthodox CI explanation, but rather supports and extends it. The implications for the role of the mind, information, and observations in QM are presented. Our Enhanced Orthodox Interpretation of Quantum Mechanics is meant to shed light on the elusive collapse of the wave function. There is the possibility that individual observers making choices in space-time by setting up quantum experiments point to the existence of the universal Observer (who should properly be designated as the Observer with capital o) with information access outside space- time, what we term NST. In future work, we will further develop the implications of these insights.

\section{Compliance with Ethical Standards}

Conflict of Interest The authors declare that they have no conflict of interest.

Open Access This article is distributed under the terms of the Creative Commons Attribution 4.0 International License (http:// creativecommons.org/licenses/by/4.0/), which permits unrestricted use, distribution, and reproduction in any medium, provided you give appropriate credit to the original author(s) and the source, provide a link to the Creative Commons license, and indicate if changes were made.

\section{References}

Atmanspacher, H. (1997). Cartesian cut, Heisenberg cut, and the concept of complexity. World Futures, 49, 333-355. https://doi.org/10.1080/ 02604027.1997.9972639.

Cassidy, D. (2008). Quantum mechanics 1925-1927: Triumph of the Copenhagen interpretation. Werner Heisenberg. NY: American Institute of Physics.

Donati, O., Missiroli, G. F., \& Pozzi, G. (1973). An experiment on electron interference. American Journal of Physics, 41, 639-644. Bibcode: 1973AmJPh..41..639D. https://doi.org/10.1119/1.1987321.

Herzog, T. J., Kwiat, P. G., Weinfurter, H., \& Zeilinger, A. (1995). Complementarity and the quantum eraser. Physical Review Letters, 75(17), 3034-3037 Bibcode:1995PhRvL..75.3034H. doi:10.1103/ PhysRevLett.75.3034. Retrieved 13 February 2014.

Kafatos, M., \& Nadeau, R. 1990(2000). The conscious universe: Parts and wholes in physical reality NY. Berlin: Springer-Verlag.

Kafatos, M., \& Narasimhan, A. (2018). The observer and access to information in the quantum universe, edit. In Jose Acacio de Barros, Carlos Montemayor, Quanta and Mind. Berlin, Heidelberg: Springer-Varlag (in press).

Kim, Y. H., Yu, R., Kulik, S. P., Shih, Y. H., \& Scully, M. A. D. (2000). "Choice" quantum eraser. Physical Review Letters, 84, 1-5. arXiv: quant-ph/9903047. Bibcode: 2000PhRvL..84...1K. https://en. wikipedia.org/wiki/Delayed_choice_quantum_eraser. https://doi. org/10.1103/PhysRevLett.84.1.

Narasimhan, A., Kafatos, M.C. (2016). Wave particle duality, the observer and retrocausality, Quantum Retrocausation III, Daniel P. Sheehan (edit) AIP Conference Proceedings, 1841:040004-1, 9. 9 pages.

Scully, M. O., \& Drühl, K. (1982). Quantum eraser: A proposed photon correlation experiment concerning observation and "delayed choice" in quantum mechanics. Physical Review A, 25(4), 22082213. Bibcode:1982PhRvA..25.2208S. https://doi.org/10.1103/ PhysRevA.25.2208.

Stapp, H. P. (2007). Mindful universe: Quantum mechanics and the participating observer (the frontiers collection) (2nd ed.). Berlin, Heidelberg: Springer-Verlag.

Stapp, H. P. (2009). Mind, matter, and quantum mechanics. Berlin, Heidelberg: Springer-Verlag.

Stapp, H. P. (2017). Quantum theory and free will: How mental intentions translate into bodily actions. Berlin, Heidelberg: Springer-Verlag.

von Neumann, J. (1995). Mathematical foundations of quantum theory. Princeton: Princeton University Press.

Walborn, S. P., et al. (2002). Double-Slit Quantum Eraser. Physical Review A, 65(3), 033818. arXiv:quant- ph/0106078. Bibcode: 2002PhRvA..65c3818W. https://doi.org/10.1103/PhysRevA.65.033818. 
Wheeler, J. A. (1981). In H. Woolf (Ed.), In Some strangeness in the proportion. Boston: Reading: Addison-Wesley Publishing Co..

Wikipedia https://en.wikipedia.org/wiki/Interpretations_of_quantum_ mechanics. Accessed April 2017

Zajonc, A. G., Wang, L. J., Zou, X. Y., \& Mandel, L. (1991). Quantum eraser. Nature, 353, 507-508. https://doi.org/10.1038/353507b0.
Zurek, W. H. (2003). Decoherence, einselection, and the quantum origins of the classical. Reviews of Modern Physics, 75(3), 715-775. 00346861/2003/75(3)/715(61)/.

Publisher's Note Springer Nature remains neutral with regard to jurisdictional claims in published maps and institutional affiliations. 